\title{
Speed Considerations Can Be of Little Concern When Outsourcing Thought to External Devices
}

\author{
Patrick P. Weis \& Eva Wiese \\ George Mason University
}

\begin{abstract}
Most research on human cognition has focused on processes "inside the box". Recently, researchers questioned this monopoly, promoting the relevance of cognitive processing "outside the box", for instance, when using a GPS to navigate. For processing that is distributed between internal and external resources to work efficiently, humans need good heuristics that help them decide when to use which resource. A novel human-computer-interaction paradigm was employed to explore whether people follow the "minimal memory" heuristic and offload cognitive processing onto external resources whenever possible or the "soft constraint" heuristic and offload cognitive processing only if it is associated with an overall higher speed than internal processing. Participants, despite lower speed, nearly exclusively cognized outside the box, which contradicts the soft constraint heuristic and mostly supports the minimal memory heuristic. Implications for human-technology interaction as well as alternative heuristics relevant for cognitive offloading are discussed.
\end{abstract}

\section{INTRODUCTION}

Humans and nonhuman primates have a long tradition in physical tool use (e.g., using a stick to reach distant objects; Vaesen, 2012). While such physical tool use might seem trivial, mastering it needs considerable neural adaptation. For example, it has been shown that when primates learn to use a stick to extend their range of actions, they integrate the stick into their internal body schema by altering somatosensory receptive fields of neurons located in the intraparietal cortex (Maravita \& Iriki, 2004). Such integration ultimately augments the primate's actual and perceived interaction abilities with the world. In other words, after including the tool into its body schema, the primate's altered receptive fields make the primate "think" differently (about the world).

But human primates have not only been augmenting their physical abilities with physical tools. They have also been augmenting their cognitive abilities with $\operatorname{cog}$ nitive tools. The historian Polybius, for instance, described cognitive tool use as early as two hundred years $\mathrm{BC}$ when describing the use of the Salamis Tablet as a counting board in ancient Greece (Menninger, 2013). These cognitive tools should, given their nature of being used for cognitive tasks, be coupled even tighter to human thought than the less cognitive physical tools.

Despite the cognitive tool's apparent relevance for the way humans think, little is known about the principles that guide human cognitive tool use. With the exponentially increasing availability of cognitive tools due to the current advances in computer technology, investigating these principles might be especially relevant. People can use dynamic notes, virtual to-do-lists, or translation engines to support their memory (Marcus, 2008). Simi- larly, navigation software can supplement spatial processing and arithmetic operations can be outsourced to calculators. It however remains unclear why people are engaging with these cognitive tools in the first place and how such engagement ultimately affects brain-based cognitive processing.

With today's ubiquity of cognitive tools, it might be tempting to consistently avoid brain-based cognitive processing in favor of external tool-based processing. Such behavior could however be problematic in several respects. For example, overly relying on occasionally malfunctioning automation during vehicle operation can lead to decreased performance (Parasuraman \& Riley, 1997) and increased likelihood of accidents (National Transportation Safety Board, 1994). It is also attentiondraining to constantly monitor a cognitive tool like a smart phone (Ward et al., 2017), which blocks internal resources that could be used otherwise. Consequently, rather than blindly relying on cognitive tools, one should aim for a balance between internal and external processing. To find that balance, heuristics about when to recruit external resources and when to better revert to internal resources would be helpful.

The goal of the current experiment is to broaden our understanding of the principles governing people's decision to use cognitive tools instead of relying on internal, brain-based resources. A novel paradigm is employed to compare two widely accepted heuristics for cognitive tool use: the "minimal memory" heuristic and the "soft constraint" heuristic. The minimal memory heuristic states that people outsource computation whenever they have the possibility to substitute neural processing and minimize neural effort (Ballard et al., 1997), whereas the soft constraint hypothesis assumes that people switch between internal and external computation to maximize 
processing efficiency as measured in time (Gray et al., 2006). To contrast both heuristics, participants perform an extended rotation task (see Shepard \& Metzler, 1971) in 1) an internal condition, where objects need to be rotated without external support, 2) an external condition, where objects need to be rotated using an external rotation knob, or 3) a choice condition, where participants can choose between internal and external rotation (i.e., participants can rely on brain-based cognitive processes or recruit a cognitive tool). The internal and external conditions are needed to measure the baseline efficiency of the respective strategy while the choice condition allows examining how these efficiencies affect the participants' decisions whether or not to use the cognitive tool. The following options regarding the choice condition are explored:

(1) If the soft constraint heuristic is followed, people will flexibly switch between internal and external cognitive processing, depending on which mode is more efficient: participants in the choice condition will prefer the locus that led to quicker answers in the forced conditions.

(2) If the minimal memory heuristic is followed, people will outsource cognitive processing whenever possible: participants in the choice condition will prefer the external to the internal locus even if internal was quicker than external processing in the forced conditions.

\section{METHODS \& MATERIALS}

\section{Participants}

Data from 150 participants was collected, 50 in each locus of cognition condition. Two participants (one in the forced external, one in the forced internal condition) were excluded because one of them pressed the wrong buttons and the other reported having briefly fallen asleep during the study. In addition, twelve participants (seven forced internal, three forced external, one choice condition) were excluded because they failed to accurately answer in at least $80 \%$ of the trials in which working and base stimulus were identical (i.e., same handedness and angle 0, see Task and Design), suggesting they did not follow task instructions. Thus, in total, 136 participants (42 forced internal, 46 forced external, 49 choice) were included in the analysis. All participants were recruited from the psychology student pool of an American university and reimbursed with research participation credits. To motivate participants, the three participants with the best performance in the rotation task were additionally rewarded with Amazon vouchers (1st place: $15 \$$ voucher; 2 nd place: $10 \$$ voucher; 3 rd place:
$5 \$$ voucher). All participants reported to be at least 18 years old, have normal or corrected to normal vision, have not been diagnosed with a psychological disorder, have no history of neurological damage (such as head injury), and not be taking any medications which affect the central nervous system. Participants provided written informed consent prior to participation. The study was approved by the local Ethics Committee.

\section{Task and Design}

To examine the interplay between internal brain-based, and external computer-supported processing, we expanded the well-established mental rotation paradigm (Shepard \& Metzler, 1971). Participants' task was to compare two stimuli, one of which has been tilted, and decide whether both stimuli are identical. In the classical paradigm, answers had to be deduced from internal processing only. In our expanded paradigm, in addition to internal processing, outsourcing mental rotation was made possible by manipulating a knob that rotates a working stimulus on the participant's computer screen. The experimental setup is illustrated in Figure 1a.

The rotation task followed a 2 × 4 × 3 design with the factors handedness, angle, and locus of rotation, respectively. The factors angle and handedness varied within participants. The factor locus of rotation varied between participants. During each trial of the rotation task, one base stimulus was presented on the right side of the screen and one working stimulus on the left side of the screen (for a prototypical trial, see Figure 1b). The working stimulus had either the same handedness as the base stimulus or a different handedness. Furthermore, the working stimulus was rotated clockwise by 0 , 60,120 , or 180 degrees (angle manipulation). In other words, the working stimulus was either the base stimulus rotated clockwise by $0,60,120$, or 180 degrees (same handedness), or the mirror image of the base stimulus rotated clockwise by $0,60,120$, or 180 degrees (different handedness condition). Participants had to indicate if the working stimulus had the same handedness as the base stimulus. To do so, participants were to press a key marked with a checkmark (same handedness) or a key marked with ' $X$ ' (different handedness). Participants had up to five seconds to provide their answer before the trial was aborted and the next trial started. Between trials, a fixation cross appeared for a time period uniformly distributed between $1500 \mathrm{~ms}$ and $2500 \mathrm{~ms}$.

Participants were assigned to groups that differed with respect to the locus of rotation: participants were either forced to solve the task using internal brain-based rotation (forced internal), were provided a rotation knob and asked to only use the rotation knob to solve the task (forced external), or were provided the same rotation 
knob and asked to decide on a trial-by-trial bases if they want to rotate internally or externally (choice). To avoid possible confounds due to proximity of the hand to the working stimulus, participants in the forced internal condition were asked to rest their free hand on the table, in proximity to where the rotation knob is located in both other conditions. Each of the twenty-four stimuli was presented in each of the four angles and in each handedness for three times, resulting in a total of 576 trials. Trials were presented in three blocks, each containing 192 trials with unique combinations of stimulus type, angle, and handedness. Within blocks, trials were randomized. Every 16 trials, participants were able to take a break and were informed about their accuracy.

\section{Stimuli}

For the rotation task, twenty-four different 2D stimuli were created using Matlab version R2015b (The Mathworks, Inc., Natick, MA; the script used for creating the stimuli is provided in Collin \& McMullen, 2002). Stimulus creation followed the Attneave procedure (Attneave $\&$ Arnoult, 1956). We created Attneave stimuli differing only in the edge parameter, ranging from three to twenty-one edges; see Figure 1.

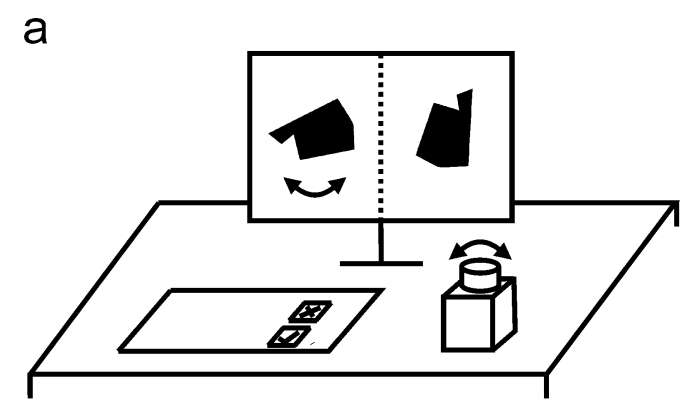

b

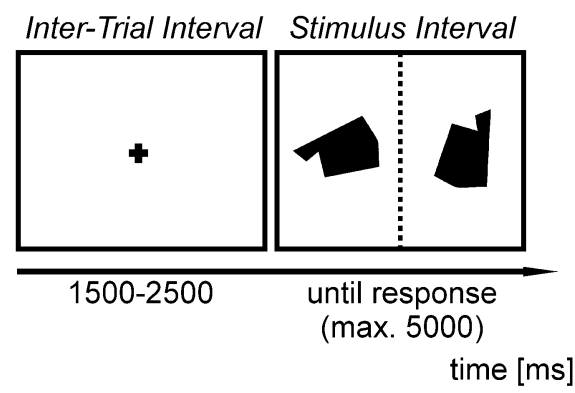

Figure 1. Extended Rotation Paradigm: (a) The experimental setup contained a computer screen for stimulus presentation, a standard keyboard to respond to the handedness question, and a rotation knob to offload cognitive processing in the forced external and choice conditions. In the forced internal condition, no rotation knob was present. (b) During each trial, a fixation cross was presented, followed by the presentation of a base on the right side and a working stimulus on the left side of the screen. Rotating the knob, if present, led to analogous rotation of the working stimulus on the screen. Participants' task was to determine if the base stimulus has the same handedness as the working stimulus. Participants could solve the task by mentally rotating one of the stimuli or by using the knob to rotate the working stimulus on the screen. For details, see Task and Design. Stimuli and devices are not drawn to scale.

\section{Procedure}

Each participant had to complete the shortened versions of three complex working memory span tasks (reading span, operation span, and symmetry span; Oswald et al., 2015), a measure of visuo-motor-coordination (computer-ported version of the rotary pursuit task; Mueller, 2012), and a custom-written rotation task. To compare the influence of general arousal between different experimental conditions, participants had to fill out the Stanford Sleepiness Scale (Hoddes et al., 1972) before and after the rotation task. In the rotation task, participants were instructed to answer as quickly and as accurately as possible. It took between 40 and 60 minutes. Overall, the study took between 75 and 100 minutes. Since it allows a more focused discussion of our main findings, working memory span and visuo-motor-coordination data will be reported elsewhere.

\section{Data Filtering and Analysis}

All trials with reaction time values above or below $2.5 \mathrm{SD}$ of the individual mean of the respective angle condition $(0,60,120$, or 180 degrees) were excluded because participants likely had used special strategies, were inattentive, or accidentally pressed too quickly during these trials, leading to an exclusion of $2.0 \%$ of all trials. To increase interpretability, all analyses were conducted on trials with in the same handedness condition only. For reaction time analyses, only correctly answered trials were used.

To test if the internal strategy is more time efficient as the external strategy, a mixed $4 \times 2$ ANOVA with the factors angle $(0,60,120,180$ degrees $)$ and locus of rotation (forced internal, forced external) was performed on participants' reaction times and followed up by onesided independent $\mathrm{t}$-tests. After identifying the more time efficient strategy, a one-sample t-tests on the externalization rate in the choice condition was employed to test if people indeed preferred the time efficient strategy. This was done in more than $50 \%$ of cases. We averaged across all non-zero angles for this analysis since we would not expect participants to externalize in the 0 angle condition. To test if externalization rate was compatible with the minimal memory heuristics, an analogue one-sample t-test was performed to test if the externalization rate at non-zero angles is different from $100 \%$. Lastly, to explore a possible speed-accuracy-tradeoff, a mixed $4 \times 2$ ANOVA with the factors angle $(0,60,120$, 180 degrees) and locus of rotation (forced internal, forced external) was performed on participant's accuracy and followed up by two-sided independent t-tests. 


\section{RESULTS}

Both angle $\left(F(3,258)=597.21, p<.001, \eta^{2}=.52\right)$ and locus of cognition $\left(F(1,86)=4.27, p=.04, \eta^{2}=.02\right)$ as well as the interaction between angle and locus of cognition $\left(F(3,258)=3.85, p=.01, \eta^{2}<.01\right)$ influenced reaction times. When forced to rotate externally, participants responded slower if base and working stimulus matched (angle $0, t(86)=2.64, p=.005$ ), deviated by 60 degrees (angle $60, t(86)=2.72, p=.004)$, or deviated by 120 degrees (angle $120, t(86)=1.82, p=.036)$. No significant differences were detected when base and working stimulus deviated by 180 degrees (angle 180, $t(86)=$ $0.61, p=0.272$ ). Reaction time data is summarized in Figure 2.

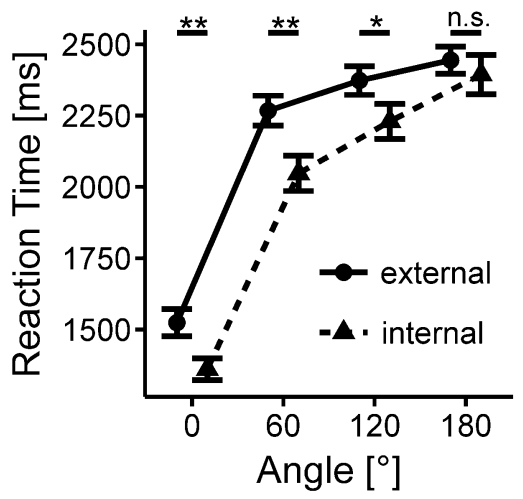

Figure 2. Reaction Time Data: The graph illustrates mean reaction time data for correctly answered trials in the forced internal and forced external conditions. Error bars represent SEM. ** p <.01, * p <.05, n.s. $\mathrm{p}>.05$

For non-zero angles $(60,120$, and 180 degrees), participants did externalize in more than $50 \%$ of trials $(M=92.4 \%, t(48)=21.38, p<.001)$ despite the associated costs in time. However, they also externalized less than $100 \%$ of trials $(t(48)=3.84, p<.001))$. Externalization data is summarized in Figure 3.

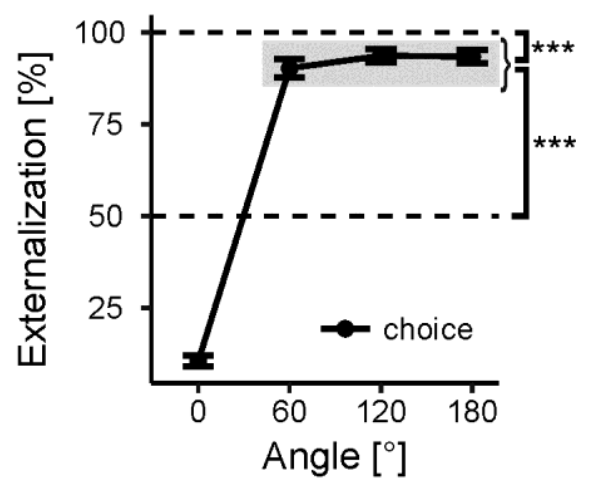

Figure 3. Externalization Data: The graph illustrates how frequently participants chose to use the external device in the choice condition. For non-zero angles (i.e., 60, 120, and 180 degrees), they used the device in more than $50 \%$ but less than $100 \%$ of trials. $* * * \mathrm{p}<.001$
Accuracy was affected by both angle $(F(3,258)=$ $\left.73.01, p<.001, \eta^{2}=.17\right)$ and locus of cognition $(F(1,86)$ $\left.=11.97, p<.001, \eta^{2}=.07\right)$ as well as the interaction between angle and locus of cognition $(F(3,258)=22.91, p$ $<.001, \eta^{2}=.05$; see Figure 4). Follow-up t-tests revealed that participants did answer less accurately in the $180^{\circ}(t(86)=5.51, p<.001)$ and $120^{\circ}(t(86)=3.10, p=$ $.002)$ conditions but not in the $60^{\circ}(t(86)=1.67, p=$ $.098)$ and $0^{\circ}(t(86)=.414, p=.680)$ conditions.

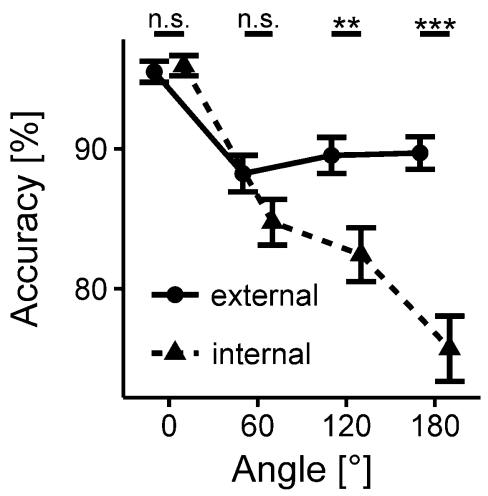

Figure 4. Accuracy Data: The graph illustrates mean accuracy data in the forced internal and forced external conditions. Error bars represent SEM. *** $\mathrm{p}<.001, * * \mathrm{p}<.01$, n.s. $\mathrm{p}>.05$

From the accuracy results, we inferred that participants might have traded speed for increased accuracy. To further explore this option, we graphed out single participant's average reaction times and accuracies of all trials with non-zero angles in the forced conditions (Figure 5a) as well as the distribution of knob use in the choice condition (Figure 5b). We additionally compared the regression lines in both forced conditions (Pearson's $r<0$ for both groups with $p<.05$; see Figure 5a). The internal regression line indicated faster answers for the whole accuracy spectrum which makes it appear unlikely that the high externalization rate in the choice condition is exclusively due to an accuracy maximization heuristic. a

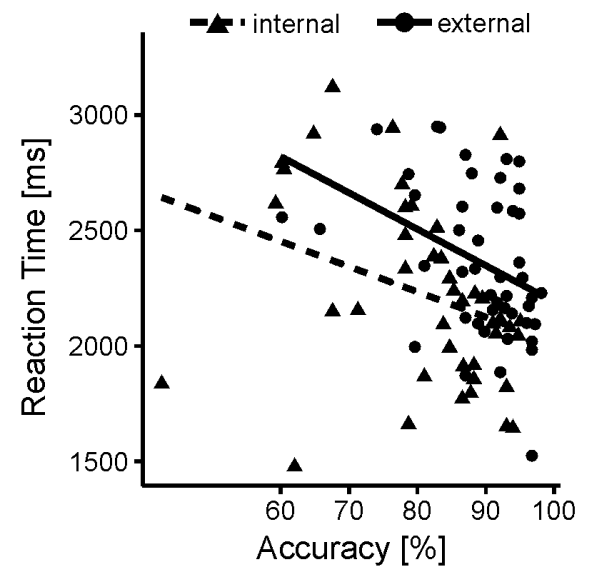

b

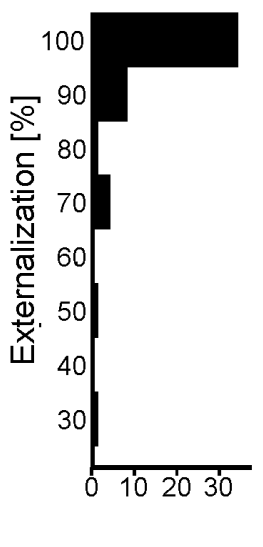

Figure 5. Single Participant Data: (a) Individual reaction time and accuracy data for the forced conditions. (b) Individual external resource use in the choice condition. Thirty-four participants used the knob in more than $95 \%$, eight in between $85 \%$ and $95 \%$, and seven in less than $85 \%$ of trials. 


\section{DISCUSSION}

In the present study, an extension of the original mental rotation paradigm was used to explore how people decide to recruit external help (i.e., a cognitive tool) when solving cognitive tasks. More specifically, the study focused on the question whether people recruit external resources as to maximize speed (soft constraint heuristic; Gray et al., 2006) or as to minimize neural load (minimal memory heuristic; Ballard et al., 1997). In the present paradigm, participants seem to strongly prefer external over internal resource use. Given that the use of the external resource was time-consuming, the strong preference for external resources is inconsistent with the soft constraint heuristic while being mostly consistent with the minimal memory heuristic. It should be noted that the two heuristics compared in this paper are by no means exhaustive but were nevertheless focused on because of their prominence in the field of cognitive offloading. It should also be noted that external resource use might depend on a person's internal capabilities, a person's expertise with the external resource, and the characteristics of the specific task, all of which were not investigated in the current study.

Whereas the present results clearly deny the primacy of time efficiency when deciding whether to use cognitive tools, they are less conclusive about whether participants tried to minimize cognitive load with their decisions to offload internal processing. At least three alternative accounts exist. Firstly, participants might have aimed at maximizing accuracy instead, even though the strong performance of some participants in the forced internal condition let this appear unlikely to be the sole reason. Secondly, participants might have had misconceptions about the external resource, e.g. they might have erroneously thought that the external resource would be more time efficient than internal processing (which would be a metacognitive bias; Dunn \& Risko, 2016). Lastly, participants in the choice condition might have been reluctant to dedicate time and resources to learn the internal strategy. They might have hesitated to go through a learning curve and use the less learning intensive external alternative. Further research is needed to disentangle those possibilities.

In conclusion, we found that people's decision to use a cognitive tool does not always primarily dependent on objective speed maximization. Even though we expected other factors to influence the decision to use a cognitive tool, we were surprised to find how comparatively irrelevant the time dimension was in the paradigm employed in the present study. Although we would not deny that humans are apt time optimizers under the right circumstances (Gray et al., 2006), the current study shows that other parameters, when present, can far outweigh the impact of time considerations. Our results imply that users are, at least in a certain margin, willing to sacrifice time, possibly in favor of other gains. On the one hand, this finding informs system designers to think holistically rather than merely focusing on time optimization. For example, users might prefer to free internal neural resources over maximizing speed. On the other hand, it informs system designers that in speed-critical systems, users might need guidance about when to recruit external resources.

\section{REFERENCES}

Attneave, F., \& Arnoult, M. D. (1956). The quantitative study of shape and pattern perception. Psychological Bulletin, 53(6), 452.

Ballard, D. H., Hayhoe, M. M., Pook, P. K., \& Rao, R. P. (1997). Deictic codes for the embodiment of cognition. Behavioral and Brain Sciences, 20(4), 723-742.

Collin, C. A., \& McMullen, P. A. (2002). Using Matlab to generate families of similar Attneave shapes. Behavior Research Methods, Instruments, \& Computers, 34(1), 55-68.

Dunn, T. L., \& Risko, E. F. (2016). Toward a Metacognitive Account of Cognitive Offloading. Cognitive Science, 40(5), 1080-1127. https://doi.org/10.1111/cogs.12273

Gray, W. D., Sims, C. R., Fu, W.-T., \& Schoelles, M. J. (2006). The soft constraints hypothesis: A rational analysis approach to resource allocation for interactive behavior. Psychological Review, 113(3), 461-482. https://doi.org/10.1037/0033-295X.113.3.461

Hoddes, E., Zarcone, V., \& Dement, W. (1972). Development and use of Stanford Sleepiness scale (SSS). Psychophysiology, 9, 150.

Maravita, A., \& Iriki, A. (2004). Tools for the body (schema). Trends in Cognitive Sciences, 8(2), 79-86. https://doi.org/10.1016/j.tics.2003.12.008

Marcus, G. (2008). What if HM had a Blackberry? Coping with amnesia, using modern technology. Psychology Today.

Menninger, K. (2013). Number Words and Number Symbols: A Cultural History of Numbers. Courier Corporation.

Mueller, S. T. (2012). PEBL: The psychology experiment building language (Version 0.10)[Computer experiment programming language]. Retrieved Nov.

National Transportation Safety Board. (1994). Stall and loss of control on final approach (Aircraft accident report No. 6308A).

Oswald, F. L., McAbee, S. T., Redick, T. S., \& Hambrick, D. Z. (2015). The development of a short domain-general measure of working memory capacity. Behavior Research Methods, 47(4), 1343-1355. https://doi.org/10.3758/s13428-014-0543-2

Parasuraman, R., \& Riley, V. (1997). Humans and automation: Use, misuse, disuse, abuse. Human Factors, 39(2), 230-253.

Shepard, R. N., \& Metzler, J. (1971). Mental rotation of threedimensional objects. Science, 171(3972), 701-703.

Vaesen, K. (2012). The cognitive bases of human tool use. Behavioral and Brain Sciences, 35(04), 203-218.

Ward, A. F., Duke, K., Gneezy, A., \& Bos, M. W. (2017). Brain Drain: The Mere Presence of One's Own Smartphone Reduces Available Cognitive Capacity. Journal of the Association for Consumer Research, 2(2), 140-154. https://doi.org/10.1086/691462 